\title{
ANALYSIS OF THE INDICATIONS AND RESULTS OF CEREBROSPINAL FLUID EXAMINATION IN CHILDREN ADMITTED TO THE PAEDIATRIC WARDS OF TWO HOSPITALS IN EASt Africa
}

\author{
G. HERBERT' ${ }^{1}$, M. NDIRITU ${ }^{2}$, R. IDRO ${ }^{2}$, J.B. MAKANI ${ }^{1}$ \\ And J. KITUNDU ${ }^{3}$
}

'Muhimbili University College of Health Sciences, P.O. Box 65000, Dar es Salaam, Tanzania

${ }^{2}$ Centre for Geographic Medicine Research-Coast, Kenya Medical Research Institute

${ }^{3}$ Department of Paediatrics and Child Health, Muhimbili National Hospital, Dar es Salaam, Tanzania

Objectives: To compare indications for lumbar puncture and findings of cerebrospinal fluid examination in children admitted at Muhimbili National Hospital (MNH) in Dar-es-salaam, Tanzania and Kilifi District Hospital (KDH) in Kenya

Study design: A descriptive, cross-sectional hospital based study.

Setting: Paediatric wards of Muhimbili National Hospital (MNH) in Dar es Salaam and Kilifi District Hospital $(\mathrm{KDH})$ in Kenya.

Results: Overall, 8,741 paediatric admissions were recorded in the two hospitals; (Muhimbili =6,228; Kilifi=2,513). Of these, 607 (6.9\%) had an LP performed; 154 out 6,228 (2.5\%) from MNH and 453 out of $2,513(18.0 \%)$ at $\mathrm{KDH}$. The most common indications for lumbar puncture at MNH were convulsions (61.4\%), neck stiffness (14.3\%) and prostration (17\%), whereas at KDH were convulsions (56.3\%), neck stiffness (7.3\%) and prostration (22.1\%). The bacterial isolates were rarely (2.3\%) obtained on culture at both hospitals. Streptococcus pneumonae and Salmonella species were the commonest causes of pyogenic meningitis.

Conclusion \& recommendations: Lumbar punctures are performed less frequently at Muhimbili than Kilifi Hospital. Pneumoccocus and Salmonella species are the commonest causes of pyogenic meningitis. Strategies should be put in place to improve the rates of lumbar puncture at MNH and the isolation of bacterial pathogens from cerebrospinal fluid cultures in both hospitals. Other possible causes for meningitis such as viruses need to be investigated.

Key words: lumbar puncture, cerebro-spinal fluid, children, infections, Tanzania, Kenya 


\section{Introduction}

A lumbar puncture (LP) is a procedure performed to collect a sample of cerebrospinal fluid (CSF) for biochemical, microbiological and cytological analysis. This technique is important in the diagnosis of a variety of infectious and non-infectious neurological conditions. The most common indication for a lumbar puncture is to collect cerebrospinal fluid in a case of suspected bacterial meningitis. Lumbar punctures are also performed to administer intrathecal medications like spinal anaesthesia.

At Muhimbili National Hospital (MNH), indications for LP have been adapted from the Integrated Management of Childhood Illnesses (IMCI) Guidelines. In neonates these include fever $\left(\geq 38.5^{\circ} \mathrm{C}\right)$ or hypothermia, bulging fontanelle (or acute increase in head circumference), high-pitched cry, irritability, lethargy, altered mental state and poor feeding. Bacterial meningitis is usually suspected in a patient ( $>3$ monthsincluding adolescents) with a history of fever and headache, photophobia, stiff neck, irritability or lethargy, vomiting and altered state of consciousness or a convulsions. In such patients, LP is indicated. Patients are usually initiated on treatment with antibiotics and antimalarials targeting suspected bacterial meningitis and severe malaria often before the $L P$ is performed. This is because of delays may be associated with processing and obtaining $L P$ results. At the Muhimbili National Hospital pathogens are isolated from very few CSF specimens. This has limited the utility of CSF results in guiding treatment among clinicians who hardly get positive results (G. Herbert, unpubl)

At Kilifi District Hospital (KDH) in coastal Kenya, LPs are indicated in patients with altered level of consciousness, history of convulsions in a child $<2$ years or prostration in children $<3$ years. It is also performed as part of septic screening in neonates. This hospital currently serves as a Centre of excellence in monitoring invasive haemophilus and pneumoccocal (bacteraemia and meningitis) in Kenya.

Acutebacterialmeningitis (ABM) isan important indication for lumbar puncture in most of hospitalised cases. ABM is associated with high mortality and neurological damage in children. In a partially prospective and retrospective review of data of children admitted to the Hospital with bacteria meningitis conducted in Ndala Mission Hospital in Tabora Tanzania in 2002 showed that the overall mortality due to bacteria meningitis was high (51\%) (Wiersinga et al., 2004).

Data on the indication for LPS and/ or CSF examination is scarce in Tanzania. Such data are important to provide information of the level of performance in management of CNS pathology and other neurological diseases especially in this era of HIV/AIDS. The objective of this study was therefore to compare indications for LPs and CSF examination results among children admitted to $\mathrm{MNH}$ and $\mathrm{KDH}$.

\section{Materials and Methods}

The Muhimbili National Hospital in Dar es Salaam, Tanzania and Kilifi District Hospital in Kenya were involved in the study. A retrospective review of patients who had LPS over a six-month period from November 2004 to April 2005 was conducted. This included all children admitted to the paediatrics wards and suspected to have central nervous system infections and had LPs performed.

Data was extracted from patient's clinical notes and laboratory registers held in both hard copies and electronic databases. At $\mathrm{MNH}$, patients

DMSJ Vol. 14 (Suppl. 1) 
who had a LP performed were identified from the laboratory registry and their clinical notes traced. A data sheet was constructed to collect the required information from patients files, microbiology and biochemistry laboratories. An electronic database was available at $\mathrm{KDH}$. An electronic request form was filled asking specifically for data on patients who had LPS during the specified period. Variables collected included: demographic characteristics such as age and sex of the patient, symptoms and signs of CNS infections such as convulsions, unconsciousness, bulging fontanelle and neck stiffness. Results of CSF laboratory examination of sugar, protein, white blood cell count and culture were recorded.

Datawere entered using Epi-Info 2002 software and Excel and analysed using version 8 of the STATA software package (Stata Corp, College Station, Texas).

\section{Results}

A total of 8,741 admissions ( $\mathrm{MNH}=6,228 ; \mathrm{KDH}=$ $2,513)$ to the paediatric wards were recorded. Of these, 607 (6.9\%) had a LP performed; 154 out 6,228 (2.5\%) from MNH and 453 out of $2,513(18.0 \%)$ in $\mathrm{KDH}$. However 42 patients from $\mathrm{MNH}$ were excluded from data analysis due to data missing (files could not be traced). Therefore a total of 565 patients from the two hospitals were included in the data analysis; 112 from $\mathrm{MNH}$ and 453 from $\mathrm{KDH}$. Most of the patients at $\mathrm{MNH}$ were females (60.7\%) whereas males (52.8\%) dominated in the number of patients admitted to $\mathrm{KDH}$.

There were more females (60.7\%) than males from $\mathrm{MNH}$ while $\mathrm{KDH}$ showed a slight male preponderance (52.8\%). Out of the total study population $50 \%$ were aged one year below

(Table 1).

Table 1: Sex distribution of patients' by age

\begin{tabular}{|llllll|}
\hline & Kilifi District Hospital & $\begin{array}{l}\text { Muhimbili } \\
\text { Hospital }\end{array}$ & \multicolumn{1}{c|}{$\begin{array}{l}\text { National } \\
\text { Proportion of patients in } \\
\text { age }\end{array}$} \\
\hline Age in months & $\mathrm{M}(\%)$ & $\mathrm{F}(\%)$ & $\mathrm{M}(\%)$ & $\mathrm{F}(\%)$ & \\
\hline $0-12$ & 53 & 46 & 61 & 50 & 50 \\
\hline $13-24$ & 17 & 20 & 20 & 18 & 19 \\
\hline $25-36$ & 13 & 11 & 11 & 12 & 12 \\
\hline $37-48$ & 8 & 9 & 5 & 12 & 9 \\
\hline$>48$ & 9 & 14 & 2 & 9 & 10 \\
\hline Total percentage & 100 & 100 & 100 & 100 & 100 \\
\hline
\end{tabular}

$M=$ male

$\mathrm{F}=$ female

The most common symptoms and indications for lumbar puncture are summarised in figure 1. Fever and convulsions were the most commonly reported symptoms.

Figure 1: Symptoms and signs in patients who had lumbar puncture performed at Muhimbili and

DMSJ Vol. 14 (Suppl. 1) 
Kilifi Hospitals

Percentage

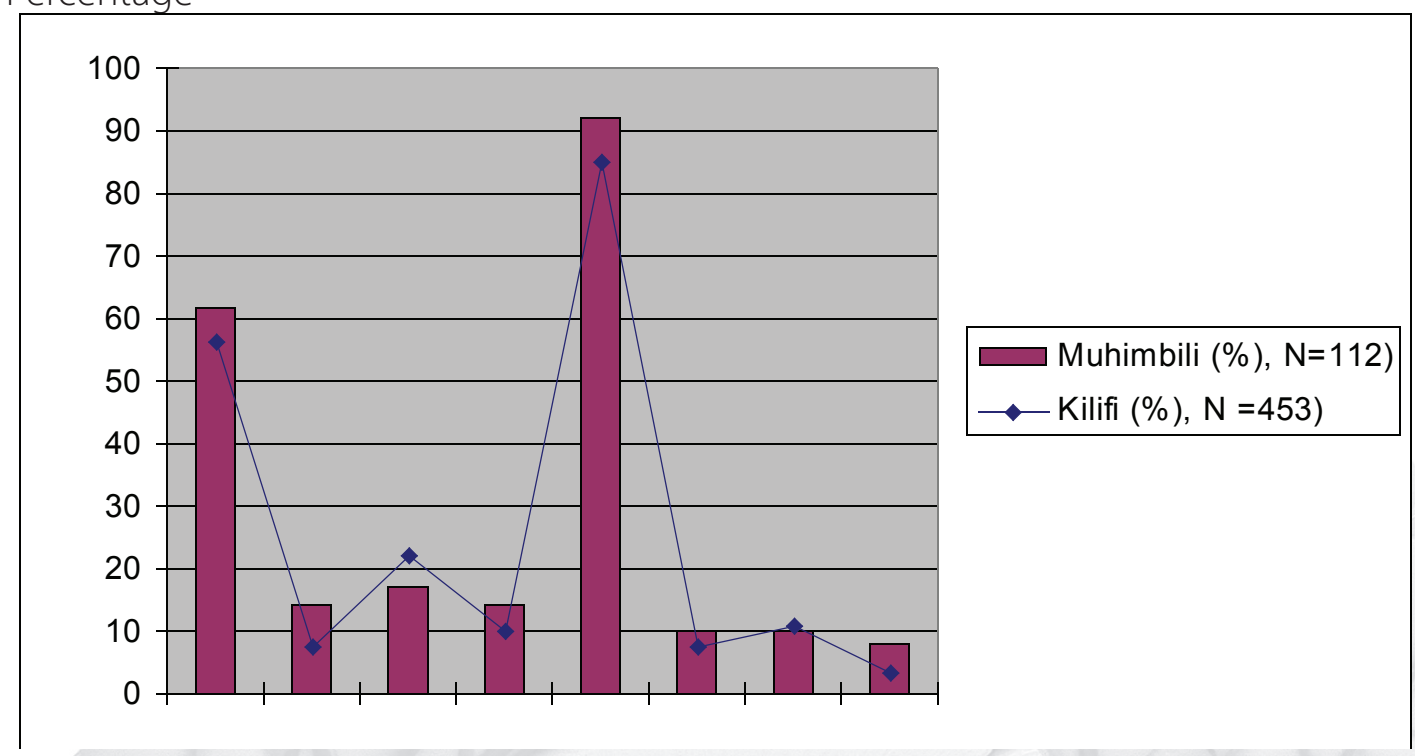

NB; child presented with one or more sign/symptom - e.g. convulsions, neck stiffness, and unconsciousness. Important differences existed between the two hospitals with regard to indications for performing a LP. KDH has its own established criteria as indications for performing a LP, a protocol that is followed by attending clinicians. MNH uses the IMCI guidelines for signs and symptoms of CNS pathology to decide on the need of performing an LP. Convulsions and prostration were the most common indication for doing LP in both hospitals.

Table 2: The levels of CSF sugar and protein in patients at Kilifi and Muhimbili Hospitals

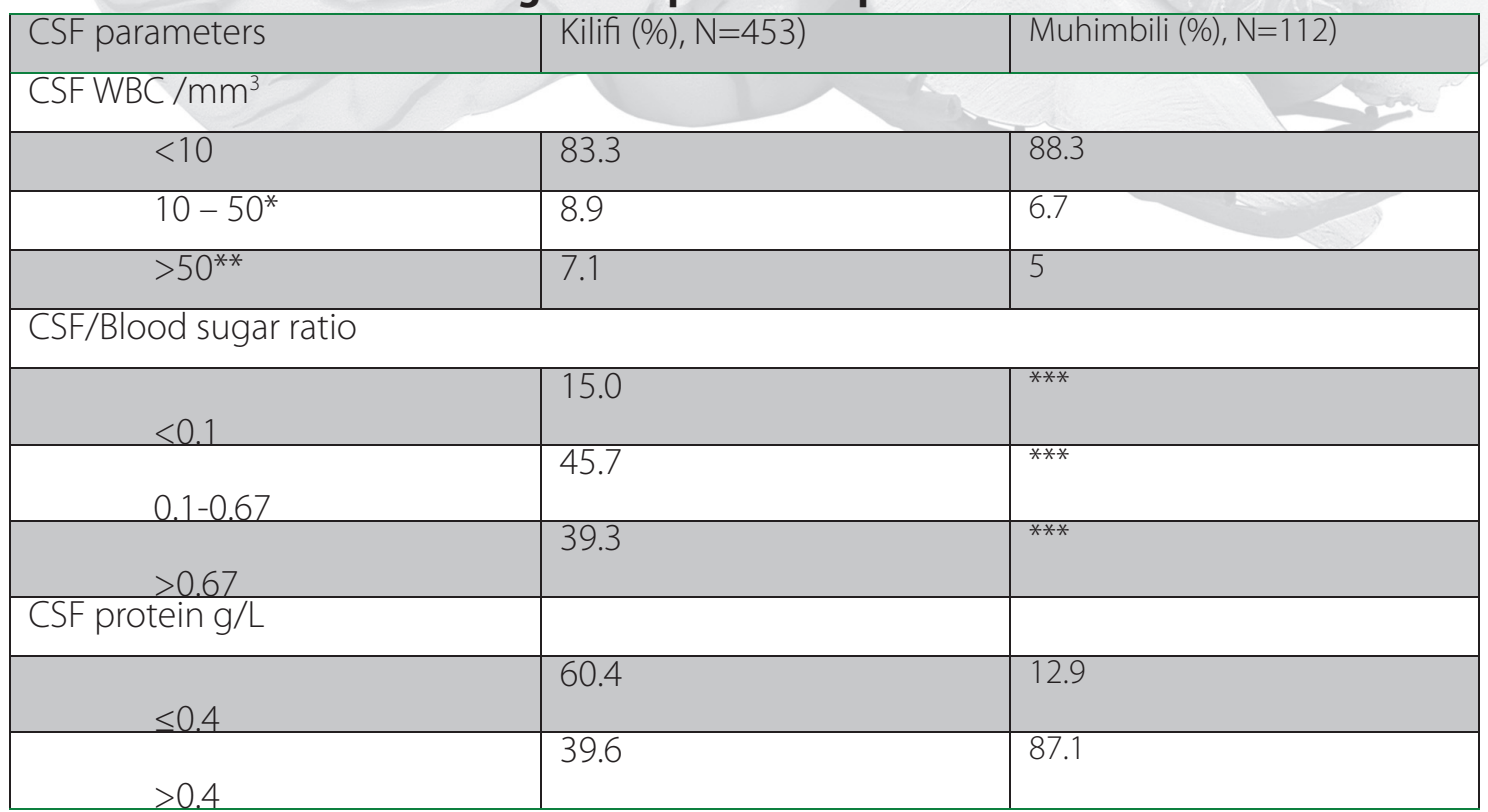

Key: * Possible meningitis; **Probable meningitis; ***blood sugar levels not determined 
Results of biochemical and cytological parameters are summarized in Table 2. There were few CSF samples collected for biochemical and cytological studies at $\mathrm{MNH}$ than at $\mathrm{KDH}$. Analysis of CSF, protein and blood sugar levels were performed in all 453 patients at $\mathrm{KDH}$. The mean CSF sugar was 3.1 (SD 1.6) $\mathrm{mmol} / \mathrm{L}$ and the median was 3.0 $\mathrm{mmol} / \mathrm{L}$. In $\mathrm{MNH}$, only CSF sugar level was performed in 86 of the 112 (77\%) patients. Blood sugar levels were not determined at the time of the LP. Therefore CSF/blood sugar ratio was not calculated from $\mathrm{MNH}$. The mean CSF sugar was 2.95 (SD 1.1) $\mathrm{mmol} / \mathrm{L}$ and the median was $3.0 \mathrm{mmol} / \mathrm{L}$. At $\mathrm{KDH}$, the majority showed normal protein levels with a mean CSF protein of 0.66 (SD 0.40) $\mathrm{g} / \mathrm{L}$ and a median of $0.3 \mathrm{~g} / \mathrm{L}$. The majority of patients from $\mathrm{MNH}$ had abnormal CSF protein (87.1 versus 39.6\%) compared to $\mathrm{KDH}$. The MNH mean CSF protein was 1.79 (SD 1.1) $\mathrm{g} / \mathrm{L}$ and the median was $1.5 \mathrm{~g} /$ $\mathrm{L}$. Bacterial isolates were rarely obtained on

culture in both hospitals. Over $97 \%$ of the samples had no isolates (Table 3).

Table 3: Common organisms isolated from CSF samples at Kilifi and Muhimbili Hospitals

\begin{tabular}{|l|l|l|l|l|}
\hline Organism & Kilifi & \multicolumn{2}{l|}{ Muhimbili } \\
\hline & $<2$ months & $>$ 2months & $<2$ months & $>$ 2months \\
\hline Acinetobacter & 1 & 0 & 0 & 0 \\
\hline Bacillus spp & 1 & 0 & 0 & 1 \\
\hline Beta haemolytic streptococcus & 1 & 0 & 0 & 0 \\
\hline Salmonella spp & 0 & 2 & 0 & 0 \\
\hline Streptococcus pneumoniae & 2 & 4 & 0 & 0 \\
\hline Streptococcus pyogenes & 1 & 0 & 0 & 0 \\
\hline Escherichia coli & 0 & 0 & 0 & 1 \\
\hline Haemophilus influenza & 0 & 0 & 0 & 1 \\
\hline No growth & 110 & 330 & 7 & 102 \\
\hline Total & 116 & 336 & 7 & 105 \\
\hline
\end{tabular}

There were differences in the isolates found in the two hospitals, with one Bacillus contaminant in both populations. Streptococcus pneumoniae and Salmonella species were the most common causes isolates in patients $>2$ months at $\mathrm{KDH}$. No pathogens were isolated in children $<2$ months at $\mathrm{MNH}$.

\section{Discussion}

Our study has shown that only a few LPs are performed among children admitted to Muhimbili National Hospital in Tanzania as compared to Kilif District Hospital in Kenya. The rate of LPS at Kilifi was seven-fold that at MNH. The availability of clear guidelines outlining the indications for

DMSJ Vol. 14 (Suppl. 1) 
LP and a mechanism for strict adherence to the protocol at $\mathrm{KDH}$ ensures that children who need a lumbar puncture get it. Moreover, the automated data management system, as well as the well-established laboratory back up for microbiological studies at Kilifi means improved accuracy in data collection. However, at Muhimbili the method of data collection and incompleteness of records are likely to have contributed to the omission of some cases during data analysis. It is however important to note that most patients at $\mathrm{MNH}$ had abnormal CSF proteins suggesting that at least patients with overt features of meningitis get a lumbar puncture.

Suspected pyogenic meningitis was the main reason to perform a LP at the two hospitals. These findings are similar to those from other studies (Wiersinga et al., 2004). None of the study participants had LPs performed for the purposes of intra-thecal medication or anaesthesia. Although some differences were observed in the organisms isolated from CSF samples at the two hospitals, it is difficult to make any conclusions given the small numbers. However, a striking observation is the absence of $\mathrm{H}$. influenzae at $\mathrm{KDH}$ and only one case at $\mathrm{MNH}$, a finding that may reflect the effectiveness of the recently introduced Hib vaccines in the childhood immunisation schedule.

In over $97 \%$ of samples submitted for microbiological analysis, no organisms were isolated. It is possible that the absence of organisms on cerebrospinal fluid culture is due to pre-treatment with antibiotics (McCarron et al., 1996). On the other hand, it is possible that many of these cases are due to viral meningitis suggesting that such infections may be a common cause of meningitis in the study area. Prospective studies should be designed to establish this.

In a recent study at $\mathrm{MNH}$ Streptococcus pneumonia, $\mathrm{C}$. neoformans and $\mathrm{H}$. influenzae type $\mathrm{B}$ were the commonest isolates in children aged $<15$ years with clinical meningitis (Matee \& Matre, 2001). Similarly, S. pneumoniae and $\mathrm{H}$. influenzae (41.9\%) were the predominant isolates among children admitted with acute bacterial meningitis between 1994 and 2000 (Mwangi et al. 2002).

Performing lumbar puncture in children is one of the useful investigations procedure for detecting CNS infections or pathology. In some hospitals, LPs are rarely performed, mainly because of the low rate of isolation of bacterial pathogens even in patients with overt features of acute bacterial meningitis. This may be due prior antibiotic therapy or poor handling of CSF specimens including delays in processing specimens. In addition, lack of proper guidelines and delays associated with waiting for LP results have contributed against its utility in guiding treatment.

In other hospitals the procedure is carried out helping to differentiate bacterial meningitis from other differential diagnoses among children who present with febrile seizures, neck stiffness, unconsciousness, symptoms and signs of complex seizures (Laditan, 1995; Berkkey et al., 2004). Sometimes presumptive treatment with antibiotics and antimalarials targeting suspected bacterial meningitis and cerebral malaria is initiated before performing LP. Such a practice avoids delays in initiating life-saving treatment and thus complications of cerebral malaria or bacterial meningitis. In a study conducted on indicators of acute

DMSJ Vol. 14 (Suppl. 1) 
bacterial meningitis in children at a rural Kenyan district, $2.0 \%$ of all admissions had the disease which was the most common indication for a LP. In this study, independent predictors form ABM were a bulging fontanelle, neck stiffness, cyanosis, impaired consciousness, partial seizures, and seizures outside the febrile convulsions age range (Kanegaye et al., 2001).

The sterilization of CSF with antibiotic pre-treatment may influence the yield from CSF and may complicate decisions on whether to continue with treatment or not. Failure to perform LPs may result in unwarranted prolonged treatment with antibiotics if the clinical presentation and other laboratory findings cannot exclude ABM.

In conclusion, lumbar punctures are performed less frequently at Muhimbili than Kilifi Hospital. Pneumoccocus and Salmonella species are the commonest causes of pyogenic meningitis. It is recommended that strategies should be put in place to improve the rates of lumbar puncture in the two hospitals and the isolation of bacterial pathogens from cerebrospinal fluid cultures. Development of clear guidelines/ protocols for lumbar punctures may be useful. Such protocols should be audited regularly.

\section{Acknowledgments}

The authors would like to express our sincere gratitude to Prof. Andrew Swai of Muhimbili National Hospital, Tanzania and Prof. Charles Newton of Kilifi District Hospital, Kenya for permission to use their clinical databases as well as using facilities. The authors are also grateful to staff in the paediatric wards of the two hospitals for their assistance and support.

\section{References}

Wiersinga, W.J.,Van dellen, Q.M., Spanjaard, L., Van Kan, H.J., Groen, A.L., Wetsteyn, J.C. (2004) High mortality among patients with bacteria meningitis in a rural hospital in Tanzania. Annals of Tropical Medicine and Parasitology 98, 271-278.

Laditan, A.A. (1995) Analysis of the results of routine lumbar puncture after a first febrile convulsion in Hofuf, Al-Hassa, Saudi Arabia. East African Medical Journal 72, 376-378.

Berkley, J.A., Versteeg, A.C., Mwangi, I., Lowe, B.S. \& Newton, C.R. (2001) Indicators of acute bacterial meningitis in children at a rural Kenyan district hospital. Pediatrics 114, 713-719.

Kanegaye, J.T., Soliemanzadeh, P. \& Bradley, J.S. (2001) Lumbar puncture in pediatric bacterial meningitis: defining the time interval for recovery of cerebrospinal fluid pathogens after parenteral antibiotic pretreatment Pediatrics 108, 1169-1174.

McCarron, B., Chaudhuri, A.K. \& Todd, A. (1996) Bacterial meningitis: the importance of cerebro-spinal fluid examination. Scottish Medical Journal 41, 12-14.

Matee, M.I. \& Matre, R. (2001) Pathogenic isolates in meningitis patients in Dar es Salaam, Tanzania. East African Medical Journal 78, 458-460.

Mwangi, I., Berkley, J., Lowe, B., Peshu, N., Marsh, K. \& Newton, C.R. (2002) Acute bacterial meningitis in children admitted to a rural Kenyan hospital: increasing antibiotic resistance and outcome. Pediatric Infectious Disease Journal 21, 1042-1048.

De Chabalier, F., Djingarey, M.H., Hassane, A. \& Chippaux, J.P. (2000) Meningitis seasonal pattern in Africa and detection of epidemics: a retrospective study in Niger, 1990-98. Transactions of the Royal Society of Tropical Medicine and Hygiene 94, 664-668.

DMSJ Vol. 14 (Suppl. 1) 